\title{
Development of an Object-Oriented Programming Tool Based on FEM for Numerical Simulation of Mineral-Slurry Transport
}

\author{
Sergio Peralta ${ }^{1}$, Jhon Cordova $^{1}$, Cesar Celis $^{1}$, and Danmer Maza ${ }^{2(\bowtie)}$ \\ ${ }^{1}$ Pontificia Universidad Católica del Perú, Lima, Peru \\ \{sergio.peralta, jcordovaa\}@pucp.pe, \\ ccelis@pucp.edu.pe \\ 2 Pontifícia Universidade Católica do Rio de Janeiro, Rio de Janeiro, Brazil \\ danmer@lmmp.mec.puc-rio.br
}

\begin{abstract}
The early stages of the development of a finite element method (FEM) based computational tool for numerically simulating mineral-slurry transport involving both Newtonian and non-Newtonian flows are described in this work. The rationale behind the conception, design and implementation of the referred object-oriented programming tool is thus initially highlighted. A particular emphasis is put on several architectural aspects accounted for and object class hierarchies defined during the development of the tool. Next one of the main modules composing the tool under development is further described. Finally, as a means of illustration, the use of the FEM based tool for simulating two-dimensional laminar flows is discussed. More specifically, canonical configurations widely studied in the past are firstly accounted for. A more practical application involving the simulation of a mineral-slurry handling device is then studied using the power-law rheological model. The results from the simulations carried out highlight the usefulness of the tool for realistically predicting the associated flow behavior. The FEM based tool discussed in this work will be used in future for carrying out high-fidelity numerical simulations of turbulent multiphase flows including fluid-particle interactions.
\end{abstract}

Keywords: Computational fluid dynamics $\cdot$ Finite element method $\cdot$ Objectoriented programming $\cdot$ Non-Newtonian fluid $\cdot$ Mineral-slurry transport

\section{Introduction}

Mining companies continuously set aside large amounts of resources to optimize their mineral extraction processes. This occurs because energy costs and greenhouse gas emissions can be significantly reduced by optimizing such processes [1]. Notice that mineral processing often involves transporting mixtures of water and/or chemical solutions carrying ground rocks known as mineral-slurries [2]. Mineral-slurry transport involves indeed multiphase flows featuring solid, liquid and gas phases. Consequently, in order to optimize mineral-slurry transporting systems, the associated complex flows need to be carefully characterized. Based on their rheological behavior, mineral-slurries may 
exhibit Newtonian or non-Newtonian rheological properties [3]. Particle size, slurry density, slurry viscosity, mass flow rate, and friction losses are thus the main design factors when designing or selecting transport slurry systems [2]. In practice both experimental or numerical techniques can be used to study and optimize mineral extraction processes [4]. Carrying out experimental tests is nevertheless relatively expensive. In contrast, numerical modeling allows performing numerical experiments at lower costs than laboratory or full-scale experiments.

Different numerical methods can be used to numerically solve the diverse multiphase flows present in practical applications. The finite element method (FEM) [5] constitutes one of the referred numerical methods. Industrial device designing and/or operating conditions optimization are some of the main FEM practical applications. In mining engineering FEM has been used for various purposes in the past [6-9]. Notice that there are several issues to be accounted for when properly developing a FEM-based computational tool. In order to obtain reliable numerical results in the shortest possible computational time for instance, FEM models need to be coded such to maximize the computational resources involved. A suitable programming paradigm should be also firstly selected before implementing a computational tool based on FEM. Objectoriented programming (OOP) has been preferred in the past for carrying out the implementation of some FEM solvers [10]. Currently there are available several commercial and open-source computational packages for FEM related applications [11-14]. Even though some of the aforementioned computational packages can be used for simulating mineral-slurry transport, it has been decided to develop a new computational tool. The main reason behind this important decision is that the new tool will include besides FEM based solvers several other numerical methods suitable for grinding processes modelling. In general, the referred new computational package is expected to be flexible enough, reducing the issues present when coupling different numerical approaches and properly modeling complex flows.

In this work, the early stages of the development of a FEM based computational tool for numerically simulating mineral-slurry transport involving both Newtonian and nonNewtonian fluids are described. Accordingly, Sect. 2 describes the rationale behind the conception, design and implementation of the referred FEM based tool. A particular emphasis is put on several architectural aspects accounted for and object class hierarchies defined during its development. One of the main modules composing the tool under development is further described in Sect. 3, including the rheological models implemented so far in the FEM based tool under development. In Sect. 4 in turn, as a means of illustration, the use of the tool for simulating two-dimensional laminar flows is discussed. More specifically, canonical configurations widely studied in the past are firstly accounted for [15-17]. A more practical application involving the simulation of a mineral-slurry flow device is then studied using a non-Newtonian fluid model. Finally, Sect. 5 summarizes the main conclusions drawn from the results obtained here.

\section{FEM Tool Development}

\subsection{Development Context}

The FEM tool discussed here constitutes one of the modules of a larger computational package under development called CFLOWSS (Complex FLOWS Solver) [18]. As its 
name indicates CFLOWSS aims to numerically solve different complex flows including mineral-slurries. Currently several Eulerian and Lagrangian approaches are being implemented in CFLOWSS. The feasibility of easily carrying out one-way or two-way coupling between different numerical approaches represents therefore one of the key features of CFLOWSS. As highlighted in Fig. 1, some of the Eulerian numerical techniques initially accounted for include finite volume methods (FVM) [19] and finite element methods (FEM) [5]. Notice that it was decided to implement a FEM module in CFLOWSS due to its proven applicability for non-Newtonian flows numerical modelling [20]. Lagrangian approaches include in turn discrete elements methods (DEM) [21] and smoothed particle hydrodynamics (SPH) [22]. Other numerical techniques such as spectral methods (SM) [23] and particle finite element methods (PFEM) [24] will be considered as well in future. Notice that the multiphysics involved when modeling complex flows justifies the inclusion of the numerical techniques indicated above.

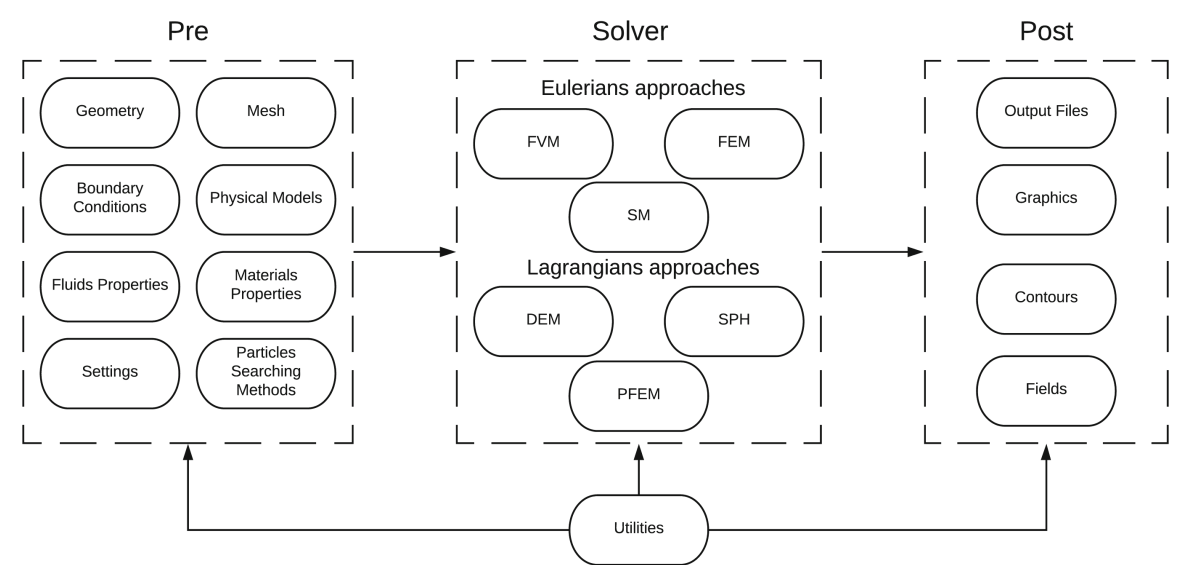

Fig. 1. Main modules composing CFLOWSS (Complex FLOWS Solver).

CFLOWSS is implemented using both an OOP paradigm and $\mathrm{C}++$ as the main programming language. The development of the CFLOWSS modules, models and numerical algorithms is continuous in order to improve the accuracy of the flow modeling processes undertaken with its aid. Three main modules compose CFLOWSS, (i) Pre, (ii) Solver and (iii) Post (Fig. 1). Geometry, mesh, physical models and other settings required for starting a simulation case are defined in the CFLOWSS Pre module. The CFLOWSS Solver module includes in turn the Eulerian and Lagrangian approaches available for the numerical simulation of complex flows. One of these numerical techniques (FEM) and its associated models and modules will be further discussed in the following sections. Finally, the CFLOWSS Post module mainly deals with the creation of results-containing output files able to be understood by well-known post-processing tools such as ParaView [25]. 


\subsection{FEM Module Class Hierarchy}

Details of the implementation of the FEM related modules in the CFLOWSS computational tool are provided in this section. Notice that the OOP paradigm used here favor the FEM implementation because FEM is essentially a modular method [26]. Many objects and abstract data have been thus extracted from the FEM formulation. As expected the FEM code implementation is based on many OOP principles such as abstraction, encapsulation, inheritance, composition and polymorphism [10]. For the sake of brevity however, only the most relevant FEM class hierarchy (Fig. 2) is discussed here. All classes shown in Fig. 2 were implemented within the CFLOWSS modules described above. In Fig. 2 the relationship between classes is represented by different line patterns. Solid lines represent inheritance. Dashed lines indicate in turn composition. Finally dash-dotted lines imply data transfer between friend classes.

The CFLOWSS utility module shown in Fig. 1 and Fig. 2 includes the main utilities required to perform a FEM simulation. For instance, the FEMArray and FEMMatrix classes included in this module allow dynamically allocating memory for the arrays and matrices utilized. FEM is essentially a numerical technique for the solution of differential and integral, linear and non-linear, equation systems [5]. Consequently, the maths involved in FEM simulations are usually complex and computationally expensive. In order to solve the associated maths then, open source mathematical libraries $[27,28]$ are used in the FEM code implemented here. The main code statements of the referred mathematical libraries are found in the FEMMathlibraries class. Numerical techniques for solving differential equations with good convergence rates such as the multigrid methods [15] will be also included in the utilities module.

Generic virtual classes typically called material classes are often used to represent material behaviors. A generic class (FEMFluid) with similar characteristics is also considered in this work. FEMFluid class is used to characterize different fluid properties such as density and molecular viscosity. In complex flow related applications, the physical models are included in the FEMmodel class. Even though this is not illustrated in Fig. 2, there is an aggregation relationship between the FEMFluid and FEMmodel classes. FEMnonNewtonianModel is a generic class that has no physical meaning, which include information and procedures common to all non-Newtonian derived models. Two wellknown non-Newtonian models, power law [16] and Carreau-Yasuda [17], have been implemented so far for simulating mineral-slurry transport. For instance, the FEMPowerLaw class is used to estimate the shear rate and the power-law apparent viscosity in a non-Newtonian power-law fluid application. The FEMTruncatedPowerLaw class involves an improvement in the power-law model achieved by using a four-parameter model [17]. In order to generate a FEM mesh, it is necessary to have a properly defined geometry and information about each element node. FEMMesh class results thus from a composition of FEMGeometry and FEMNode classes. Notice that FEMGeometry class allows setting the geometric configuration accounted for in a FEM simulation, whereas FEMNode class is used to store nodal information. FEMBoundaryConditions class is used in turn to set the governing equations boundary conditions (BC). Configurable BC include Dirichlet and Neumman BC [5]. Finally, FEMBasicFunctions allows configuring the FEM interpolation functions on each element. 


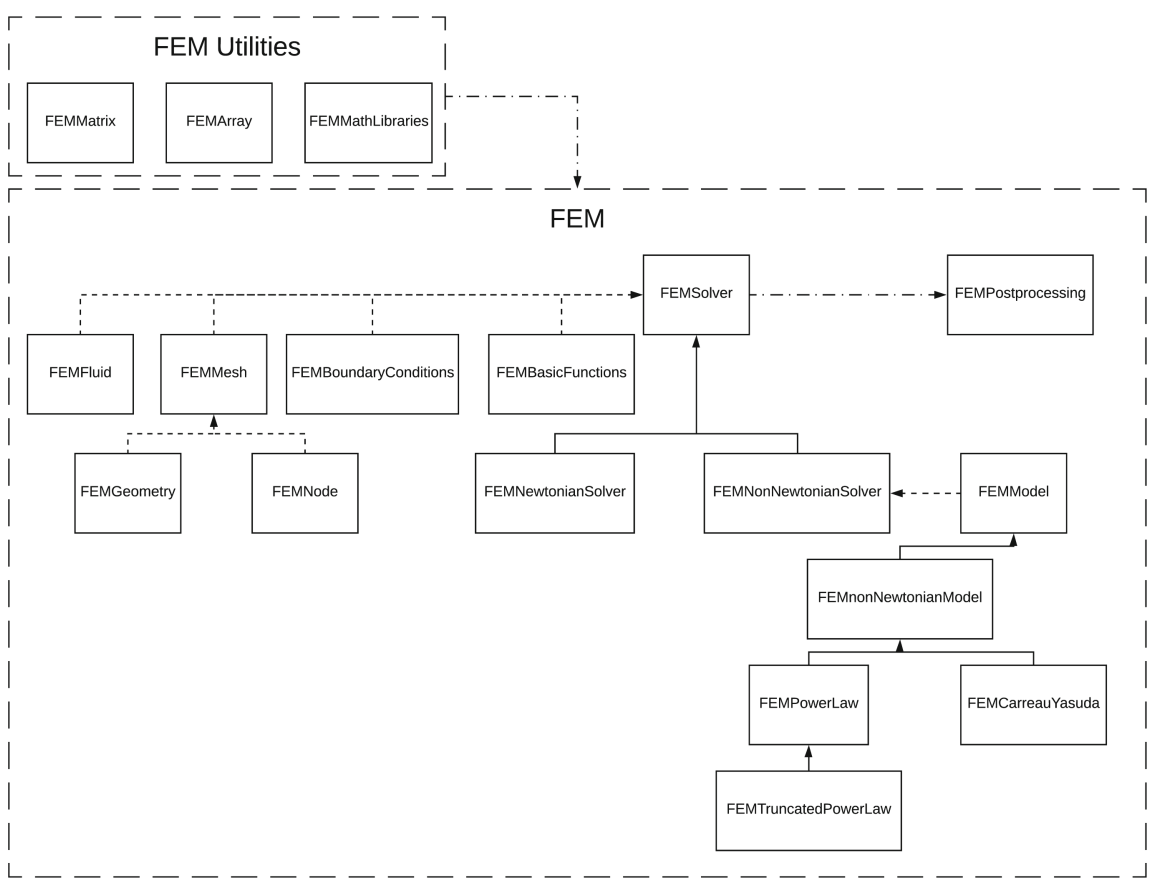

Fig. 2. FEM module class hierarchy in CFLOWSS.

The main class of the CFLOWSS FEM module is the FEMSolver class. This class is composed of FEMFluid, FEMMesh, FEMBoundaryConditions and FEMBasicFunctions classes. That is, the FEMSolver class instantiates objects of these classes and accesses through these objects all methods included in such classes. Furthermore, the FEMSolver class has also access to all mathematical algorithms related classes required to solve any FEM simulation. Non-linear equations systems for instance are linearized and then solved with numerical methods such as the Newton-Raphson one [5]. Both FEMNewtonianSolver and FEMNonNewtonianSolver classes are inherited from the FEMSolver one (Fig. 2). The FEMnonNewtonianModel generic class composes the latter class. Finally, results related data coming out from the FEMSolver class are sent to the FEMPostprocessing class for further post-processing.

\section{FEM Formulation}

\subsection{Finite Element Method Approach}

In the CFLOWSS FEM module under discussion here, the particular approach used for solving the flow governing equations is the weighted residuals method [5]. In general, for a domain $\Omega$, the weighted residuals formulation implies solving a equation of the form, 


$$
\int_{\Omega} w R \mathrm{~d} \Omega=0,
$$

where $w$ is a subspace of weighted functions and $R$ is the residual between the differential equation exact solution and a finite-dimension approximated solution [29]. The weighted residuals method receives different names according to its basis and weighted functions. When the space of weighted functions is identical to the space of the basis ones, the weighted residuals method is called Galerkin method. The Galerkin method is utilized in the CFLOWSS FEM module because of its implementation advantages when compared to other similar methods [29].

\subsection{Galerkin Method}

In order to illustrate the application of the Galerkin method, two-dimensional, laminar, incompressible flows are accounted for here. For such flows the mass and momentum transport equations are expressed as [30],

$$
\begin{gathered}
\nabla \cdot \boldsymbol{u}=0, \\
\rho \boldsymbol{u} \cdot \nabla \boldsymbol{u}=\nabla \cdot \mathbf{T},
\end{gathered}
$$

where $\rho$ stands for fluid density and $\boldsymbol{u}$ is the absolute velocity vector. In addition, for Newtonian fluids, the total stress tensor $\mathbf{T}$ is computed according to,

$$
\mathbf{T}=-p \mathbf{I}+\mu\left[\nabla \boldsymbol{u}+(\nabla \boldsymbol{u})^{\boldsymbol{T}}\right]
$$

In Eq. (4), $p$ represents the absolute pressure and $\mu$ the fluid dynamic viscosity.

The application of the Galerkin method to a flow governed by the transport equations highlighted above leads to $x$ and $y$-axis momentum equations of the form,

$$
\begin{aligned}
R_{m x}^{i} & =\int_{\Omega} \rho \psi_{i}\left[u \frac{\partial u}{\partial x}+v \frac{\partial u}{\partial y}\right]+\frac{\partial \psi_{i}}{\partial x}\left[-p+2 \mu \frac{\partial u}{\partial x}\right] \\
& +\frac{\partial \psi_{i}}{\partial y}\left[\mu\left(\frac{\partial u}{\partial y}+\frac{\partial v}{\partial x}\right)\right] \mathrm{d} \Omega-\int_{\Gamma} \psi_{i} f_{x} \mathrm{~d} \Gamma=0 i=1, \ldots, N \\
R_{m y}^{i} & =\int_{\Omega} \rho \psi_{i}\left[u \frac{\partial v}{\partial x}+v \frac{\partial v}{\partial y}\right]+\frac{\partial \psi_{i}}{\partial y}\left[-p+2 \mu \frac{\partial v}{\partial y}\right] \\
& +\frac{\partial \psi_{i}}{\partial x}\left[\mu\left(\frac{\partial u}{\partial y}+\frac{\partial v}{\partial x}\right)\right] \mathrm{d} \Omega-\int_{\Gamma} \psi_{i} f_{y} \mathrm{~d} \Gamma=0 i=1, \ldots, N .
\end{aligned}
$$


In these equations, $\psi_{i}$ are the weighted functions related to the momentum equations, which are equivalent to the basis ones. Biquadratic functions have been taken into account for the basis functions implementation in the CFLOWSS FEM module. $N$ here is the number of algebraic momentum equations. Notice that the line integrals $\int_{\Gamma} \psi_{i} f \mathrm{~d} \Gamma$ appearing in Eqs. (5) and (6) allow imposing boundary conditions on the domain $\Omega$. The mass transport equation reads in turn as follows,

$$
R_{c}^{i}=\int_{\Omega}\left(\frac{\partial u}{\partial x}+\frac{\partial v}{\partial y}\right) \chi_{i} \mathrm{~d} \Omega=0 i=1, \ldots, M
$$

where $\chi_{i}$ are the weighted functions related to the continuity equations. Linear functions have been accounted for in this case. $M$ represents here the number of algebraic equations related to continuity. In order to complement the FEM modeling detailed here, some rheological models implemented in the CFLOWSS FEM based tool for simulating non-Newtonian flows are described in the following section.

\subsection{Rheological Modeling of Non-Newtonian Flows}

Mineral slurries rheological properties vary according to its solids concentration. When the mineral slurries' solids concentration increases, the associated apparent viscosity $\eta$ is no longer constant but depends on shear stress $\tau$ and/or shear rate $\dot{\gamma}$ [3]. For nonNewtonian flows related applications, the dynamic viscosity $\mu$ appearing in Eqs. (5) and (6) has to be replaced with a term of the form $\eta(\dot{\gamma})$. In this work, the $\dot{\gamma}$ magnitude is calculated locally using the second invariant of the rate-of-strain tensor [16]. The associated components of the rate-of-strain tensor are estimated in turn from the local velocity field [31]. Three typical models commonly used to compute the $\eta(\dot{\gamma})$ term are included in the CFLOWSS FEM based tool.

\section{Power-Law Model}

The simplest representation of the apparent dynamic viscosity of a non-Newtonian fluid is given by the power-law model [16],

$$
\eta(\dot{\gamma})=K \dot{\gamma}^{n-1}
$$

where $K$ and $n$ are the empirically obtained consistency and flow behavior indexes, respectively. Notice that this model may present some issues at relatively low and high shear rates, where additional model parameters are required to properly characterize the non-Newtonian fluids' behavior [17]. The power-law model can be used then with caution in non-Newtonian flow applications such as mineral slurries [3].

\section{Carreau-Yasuda Model}

The Carreau-Yasuda model is a five-parameter model used when there are large deviations from the power-law one at relatively low and high shear rates [16]. NonNewtonian rheological properties of flows carrying solid particles such as mineral 
slurries may be described indeed by the Carreau-Yasuda model [3]. In this model, the apparent dynamic viscosity is estimated from,

$$
\eta(\dot{\gamma})=\mu_{\infty}+\left(\mu_{0}-\mu_{\infty}\right)\left(1+(\lambda \dot{\gamma})^{a}\right)^{\frac{n-1}{a}}
$$

Where $\mu_{0}$ is the zero-shear rate viscosity, $\mu_{\infty}$ the infinite-shear-rate viscosity, $\lambda$ the fluid relaxation time and $a$ is a dimensionless parameter describing the width of the transition region between the zero-shear-rate region and the power-law one [32].

\section{Truncated Power-Law Model}

The four-parameter truncated power-law model represents in turn an improvement on the pure power-law one for all shear rate range [17]. The apparent dynamic viscosity is computed in this case as,

$$
\eta(\dot{\gamma})= \begin{cases}\mu_{0}, & \dot{\gamma}<\dot{\gamma}_{1} \\ K \dot{\gamma}^{n-1}, & \dot{\gamma}_{1}<\dot{\gamma}<\dot{\gamma}_{2} \\ \mu_{\infty}, & \dot{\gamma}_{2}<\dot{\gamma}\end{cases}
$$

In this model, the shear rates at which the low- and high-viscosity cut-offs are introduced are estimated from, respectively,

$$
\dot{\gamma}_{1}=\left(\frac{K}{\mu_{0}}\right)^{\frac{1}{1-n}}, \quad \dot{\gamma}_{2}=\left(\frac{K}{\mu_{\infty}}\right)^{\frac{1}{1-n}} .
$$

Some results obtained from the use of the CFLOWSS FEM based tool accounting for these different rheological models are described in the following section.

\section{Results and Discussion}

The main results obtained from the simulation of two-dimensional laminar flows using the CFLOWSS FEM based tool are discussed in this section. Canonical configurations widely studied in the past are firstly accounted for, followed by a practical application involving the simulation of a mineral-slurry flow device and non-Newtonian flows.

\subsection{Newtonian Laminar Flow Between Infinite Parallel Plates}

The fully-developed two-dimensional Newtonian laminar incompressible steady-state flow between two infinite parallel plates is firstly considered here. The associated geometry accounted for is schematically shown in Fig. 3(a). The analytical solution characterizing this particular flow was obtained from Wang's work [33].

Velocity fields computed using thirty different meshes (ranging from 2 to 1800 elements) were initially compared with their corresponding analytical solution and the obtained average root-mean-square error (RMSE) was about 4.9E-08. For illustrative purposes, velocity profiles computed using a 1250-elements mesh and analytically are compared in Fig. 3(b). As noticed from this plot, the numerical and analytical results agree relatively well. 


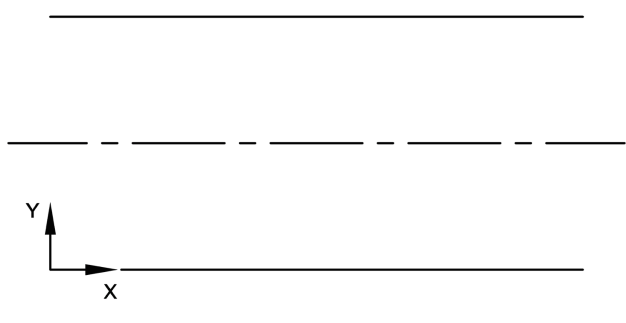

(a)

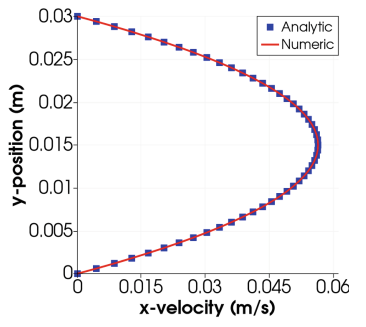

(b)

Fig. 3. (a) Geometry accounted for when computing Newtonian laminar flows between parallel plates. (b) x-velocity profiles obtained analytically and using the CFLOWSS FEM based tool.

\subsection{Lid-Driven Square Cavity}

The lid-driven cavity configuration has been widely utilized in the past as a standard verification case for new CFD codes [34]. This configuration has been also used here for verifying the CFLOWSS FEM based tool accounting for a Newtonian flow. The numerical results discussed in this section have been compared with those obtained by Ghia et al. [15] at Reynolds numbers of 100 and 400. Accordingly, Fig. 4(a) shows RMSE based mesh independency results involving the $\mathrm{u}$ and $\mathrm{v}$ velocity components along the vertical and horizontal lines passing through the cavity geometric center. These results indicate in particular that when the mesh has more than 200 elements, the RMSE values remain almost constant and less than $5 \%$.

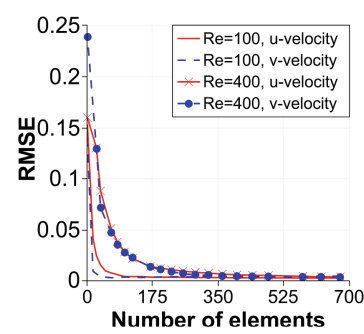

(a)

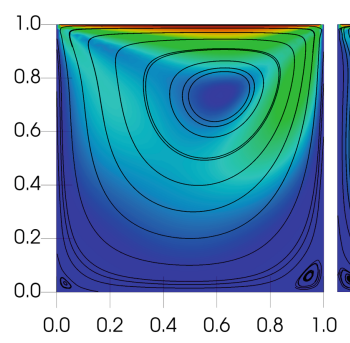

(b)

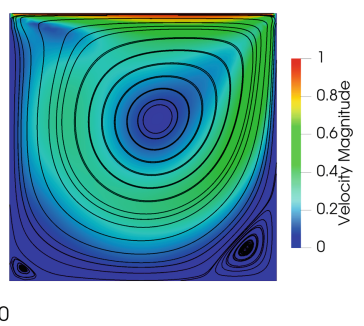

(c)

Fig. 4. (a) RMSE vs number of mesh elements. Lid-driven cavity velocity contours and streamline patterns computed by the CFLOWSS FEM based tool for (b) $\mathrm{Re}=100$ and (c) $\operatorname{Re}=400$.

The lid-driven cavity velocity contours and streamline patterns computed by the CFLOWSS FEM based tool for Reynolds numbers of 100 and 400 are shown in Fig. 4(b) and (c), respectively. A 676-elements mesh with an aspect ratio equal to one was utilized for obtaining these results, which are comparable to those obtained by Ghia et al. [15]. In particular, the streamline patterns included in these plots reveal that the main eddy center moves towards the cavity geometric center as Reynolds number increases [34]. 
The growth of secondary eddies (located at the cavity bottom left and right regions) is also observed in Fig. 4(b) and (c) as Reynolds number increases. In addition, Fig. 5 shows uvelocities along the vertical line and v-velocities along the horizontal line passing through the square cavity geometric center for Reynolds numbers of 100 and 400 . The numerical results obtained here are compared in this figure to those obtained by Ghia et al. [15]. As noticed from Fig. 5 the agreement between the CFLOWSS FEM based tool and the Ghia et al. results is quite good. The average RMSE values for the Reynolds numbers of 100 and 400 is indeed about 0.0027 and 0.036 , respectively.

\subsection{Non-Newtonian Laminar Flow Between Infinite Parallel Plates}

For the same considerations described in Sect. 4.1, in order to verify the CFLOWSS FEM based tool capabilities for dealing with non-linear fluid viscosities, a non-Newtonian flow passing through two infinite parallel plates has been simulated. The non-Newtonian flow velocity profiles computed then using the Carreau-Yasuda, power-law and truncated power-law models are shown in Fig. 6. When possible, the numerical results obtained here are compared with their respective analytical solutions $[16,17]$. Notice that for the Carreau-Yasuda model there is no analytical solution available [32].

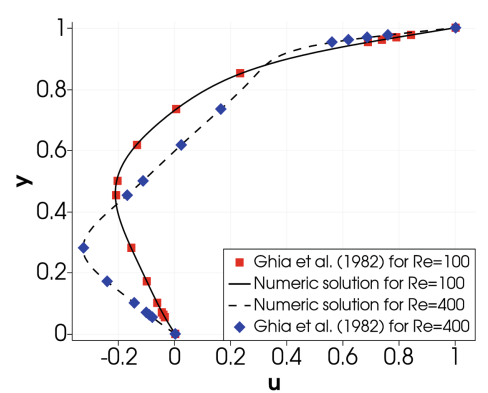

(a)

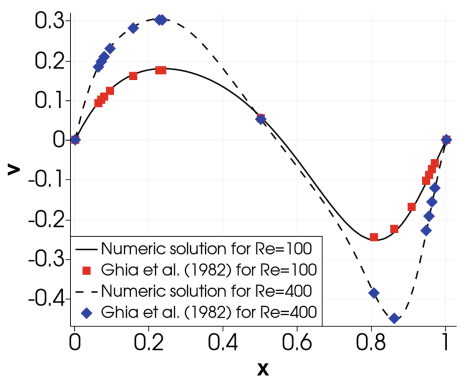

(b)

Fig. 5. (a) u-velocities along the vertical line and (b) v-velocities along the horizontal line passing through the square cavity geometric center for both $\operatorname{Re}=100$ and $\operatorname{Re}=400$.

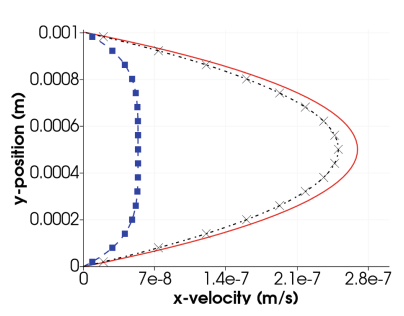

(a)

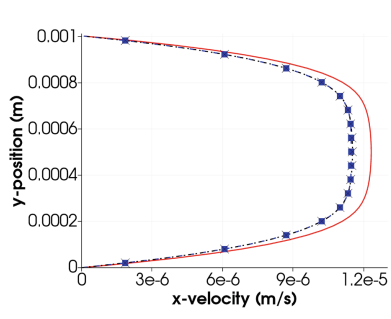

(b)

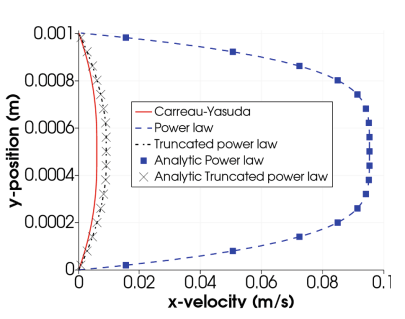

(c)

Fig. 6. Non-Newtonian flow velocity profiles obtained using Carreau-Yasuda, power-law and truncated power-law models at different pressure gradients. (a) $1 \mathrm{~Pa} / \mathrm{m}$, (b) $5 \mathrm{~Pa} / \mathrm{m}$, (c) $75 \mathrm{~Pa} / \mathrm{m}$. 
In addition, the fluid properties and geometric configuration simulated in this case were extracted from [17], accounting for a density value of $1 \mathrm{~kg} / \mathrm{m}^{3}$. The main results indicate that, accounting for a $50 \times 25$ elements mesh, RMSE values of $2.4 \mathrm{E}-23$ and 3.7E-13 in Fig. 6(a), 5.6E-09 and 2.7E-10 in Fig. 6(b), and 2.6E-07 and 6.6E-7 in Fig. 6(c) characterize the truncated power-law and power-law models, respectively. Moreover, the influence of the pressure gradient values on the velocity profiles associated with each rheological model included in Fig. 6 is similar to that described in literature [17].

\subsection{Slurry Receiving Chamber}

The receiving chamber of a slurry distribution box [35] has been simulated as well using the CFLOWSS FEM based tool as an example of the engineering situations where this tool can be applied. It is worth noticing that turbulent models for multiphase flows including fluid-particle interactions will be implemented later on in the CFLOWSS FEM based tool so they are not currently available yet. Even so, currently this tool can produce reliable results for some operating conditions characterizing mineral slurryhandling devices. For instance, when the mineral-slurry both features relatively low flow velocities and contains large amounts of solid particles, the mineral-slurry transport may be considered as a laminar non-Newtonian flow [36]. The referred situation can be modeled using the current version of the CFLOWSS FEM based tool under discussion here. In particular, different flow patterns, velocity fields and geometric configurations associated with the associated slurry-handling devices can be assessed using it. Accordingly, a particular geometric configuration of a slurry receiving chamber has been accounted for in this work as shown in Fig. 7(a). Notice from this figure that the vertical distance of the mineral-slurry exit measured from the device bottom is denoted by $\mathrm{Y}$. This vertical distance has been varied in the numerical simulations carried out here for flow pattern analyses. All results discussed in this section have been obtained accounting for a non-Newtonian power-law model, and mineral-slurry related parameters reading as follows, (i) $\rho=1370 \mathrm{~kg} / \mathrm{m}^{3}$, (ii) $\mu=1.6 \mathrm{~Pa} . \mathrm{s}$ and (iii) $n=0.4$ [16].

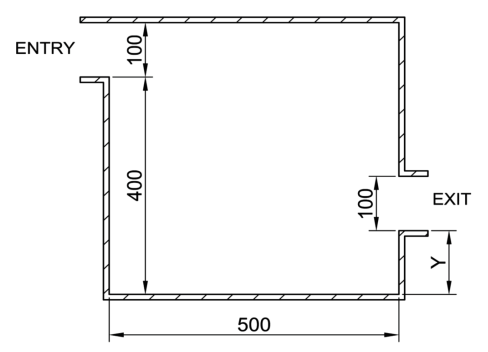

(a)

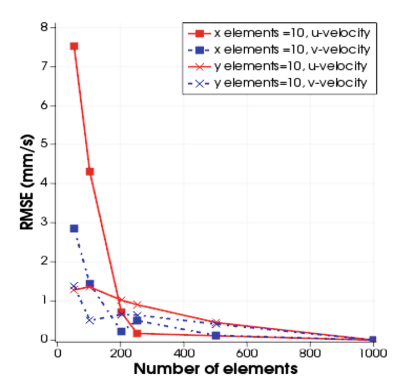

(b)

Fig. 7. (a) Slurry receiving chamber geometric configuration (units in mm). (b) RMSE versus number of mesh elements. 
A mesh-independency assessment has been initially carried out as shown in Fig. 7 (b), accounting for a 1000 elements mesh as the reference mesh for computing the RMSE values. Several numerical simulations were so performed gradually increasing the number of mesh elements along one axis and keeping constant this number along the other one, i.e., varying the mesh elements aspect ratio. For instance, the squaresymbols curves in Fig. 7(b) were obtained from simulations where the number of mesh elements along the $\mathrm{x}$-axis was constant and equal to 10 . Similarly, the $\times$-symbols curves correspond to simulations where the number of mesh elements along the $y$-axis was equal to 10 . Notice that the RMSE values of the two velocity components were accounted for in this initial verification stage. The results shown in Fig. 7(b) indicate in particular when the mesh has more than 500 elements, the RMSE values (lower than $0.5 \mathrm{~mm} / \mathrm{s}$ ) no longer vary significantly.

The main results obtained from the numerical simulations of the slurry receiving chamber accounted for here are summarized in Fig. 8. In particular, Fig. 8(a) to (c) and Fig. 8(d) to (f) show, respectively, the streamlines and velocity contours characterizing pure Newtonian $(n=1.0)$ and non-Newtonian mineral-slurry $(n=0.4)$ flows passing through the receiving chamber when varying the flow exit location. For obtaining these results, a constant velocity profile at the receiving chamber entry of $75 \mathrm{~mm} / \mathrm{s}$ was initially imposed. The referred results highlight in particular that both velocity fields and eddy sizes are influenced by the fluid's rheological properties and flow exit locations. When increasing $\mathrm{Y}$ indeed, progressively larger eddies are formed in the chamber bottom left corner and those in the top right one almost disappear. Properly locating these eddies is important because sedimentation is fostered in eddiescontaining regions.

Pressure drops as a function of the flow exit location ( $\mathrm{Y}$ value), the flow entry velocity and the fluid rheological properties were also studied as illustrated in Fig. $8(\mathrm{~g})$. This plot shows firstly that for the three imposed entry velocities, the highest pressure drops are obtained at the flow exit relative locations of 0 and $1, Y=0$ and $\mathrm{Y}=400 \mathrm{~mm}$, respectively. In addition, for relatively low flow entry velocities $(\approx 25$ $\mathrm{mm} / \mathrm{s})$, compared to the Newtonian case $(\mathrm{n}=1)$, the mineral-slurry flow $(\mathrm{n}=0.4)$ presents higher pressure drops. This finding comes from the use of the power-law model, which considerably increases viscosity at low velocity gradients. This last aspect can be confirmed by analyzing the results shown in Fig. 8(h), which shows the relationship between fluid's rheological properties and pressure drops for different flow entry velocities at one single flow exit location, $\mathrm{Y}=250 \mathrm{~mm}$. In accordance with the results shown in Fig. $8(\mathrm{~h})$, at relative high flow entry velocities $(\approx 75 \mathrm{~mm} / \mathrm{s})$, relatively higher pressure drops characterize Newtonian flows (Fig. 8(g)). Following these results, slurry-handling devices designers can define proper device configurations according to their requirements. 
(a)

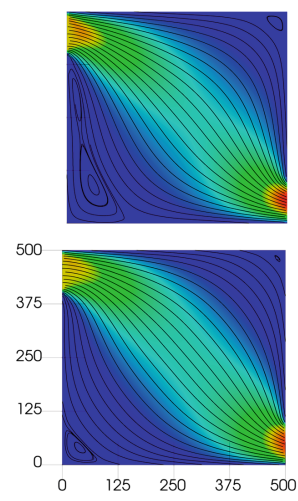

(d) (b)
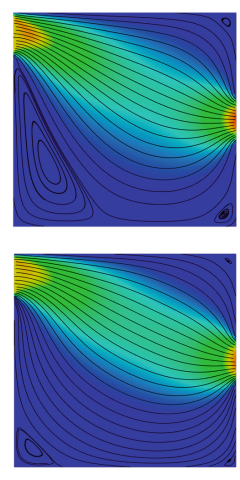

(e) (c)
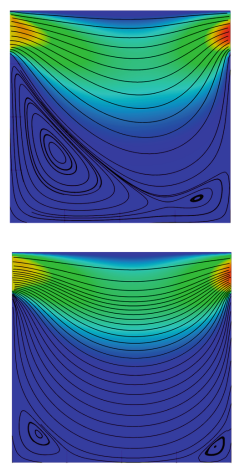

(f)

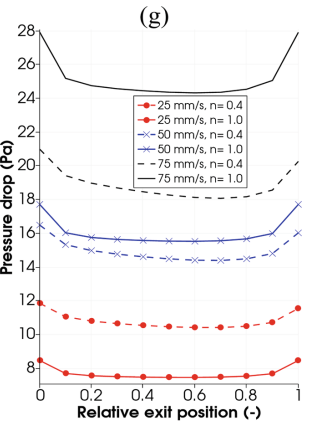

(h)

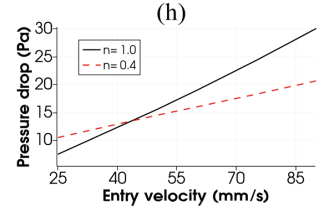

Fig. 8. Velocity contours and streamline patterns characterizing Newtonian $(n=1.0)((a)$ to $(c))$ and mineral-slurry $(\mathrm{n}=0.4)$ flows passing through the receiving chamber for exit positions $\mathrm{Y}$ of $0 \mathrm{~mm}$ ((a), (d)), $200 \mathrm{~mm}$ ((b), (e)) and $400 \mathrm{~mm}$ ((c), (f)). (g) Pressure drop versus relative exit position for different imposed inlet velocities and rheological properties. (h) Pressure drop versus receiving chamber entry velocity for Newtonian and mineral-slurry flows at $\mathrm{Y}=250 \mathrm{~mm}$.

\section{Conclusions}

In this work, the early stages of the development of a FEM based computational tool for numerically simulating mineral-slurries transport involving both Newtonian and non-Newtonian flows were described. The rationale behind the conception, design and implementation of the referred FEM based tool was thus initially described. Several architectural aspects accounted for and object class hierarchies defined during its development were detailed next. The particular approach used for solving the flow governing equations, i.e., the Galerkin method, a variation of the weighted residuals one, was emphasized as well, along with the non-Newtonian flow models implemented in the referred tool for dealing with mineral-slurries rheological aspects. As a means of illustration, the FEM based computational tool discussed in this work was used for studying two-dimensional, laminar, incompressible flows. Canonical configurations studied in the past were firstly accounted for, followed by a practical application involving the simulation of a mineral-slurry handling device and non-Newtonian flows. When possible, the numerical results obtained here were compared with analytical solutions and data available in literature and the corresponding agreement was relatively good. It is concluded therefore that the FEM based tool is useful for realistically predicting the associated flow behavior. Once fully developed, the computational tool discussed in this work will be used for carrying out high-fidelity numerical simulations of turbulent multiphase flows including fluid-particle interactions. 
Acknowledgements. This work has been funded by CONCYTEC-FONDECYT (Peru) within framework E041-01, Contract No. 155-2018-FONDECYT-BM-IADT-AV.

\section{References}

1. Levesque, M., Millar, D., Paraszczak, J.: Energy and mining-the home truths. J. Clean. Prod. 84, 233-255 (2014)

2. Mular, A.L., Halbe, D.N., Barratt, D.J. (eds.): Mineral Processing Plant Design, Practice, and Control: Proceedings, vol. 1. SME, Denver (2002)

3. Michaelides, E., Crowe, C.T., Schwarzkopf, J.D.: Multiphase Flow Handbook. CRC Press, Boca Raton (2016)

4. Xu, G., et al.: Computational fluid dynamics applied to mining engineering: a review. Int. J. Mining Reclam. Environ. 31(4), 251-275 (2017)

5. Reddy, J.N., Gartling, D.K.: The Finite Element Method in Heat Transfer and Fluid Dynamics. CRC Press, Boca Raton (2010)

6. Han, P., et al.: FEM modeling on the compaction of Fe and Al composite powders. J. Mining Metall. B: Metall. 51(2), 163-171 (2015)

7. Pater, Z., Bartnicki, J., Kazanecki, J.: 3D finite elements method (FEM) analysis of basic process parameters in rotary piercing mill. Metalurgija 51(4), 501-504 (2012)

8. Kovačević, D., et al.: Optimal finite elements method (FEM) model for the jib structure of a waterway dredger. Metalurgija-Zagreb 51(1), 113 (2012)

9. Kocich, R., Kliber, J., Herout, M.: Finite elements method (FEM) simulation based prediction of deformation and temperature at rolling of tubes on a pilgrim mill. Metalurgija 48(4), 267-271 (2009)

10. Bittencourt, M.L.: Using C++ templates to implement finite element classes. Eng. Comput. 17(7), 775-788 (2000)

11. COMSOL Multiphysics. https://www.comsol.com

12. ANSYS. https://www.ansys.com

13. Deal.II. https://www.dealii.org/

14. ELMER. https://www.csc.fi/web/elmer

15. Ghia, U.K.N.G., Ghia, K.N., Shin, C.T.: High-resolutions for incompressible flow using the Navier-Stokes equations and a multigrid method. J. Comput. Phys. 48(3), 387-411 (1982)

16. Chhabra, R.P., Richardson, J.F.: Non-Newtonian Flow and Applied Rheology: Engineering Applications. Butterworth-Heinemann, Oxford (2011)

17. Lavrov, A.: Flow of truncated power-law fluid between parallel walls for hydraulic fracturing applications. J. Non-Newtonian Fluid Mech. 223, 141-146 (2015)

18. Angeles, L., Celis, C.: Assessment of neighbor particles searching methods for discrete element method (DEM) based simulations. In: VI International Conference on ParticleBased Methods - Fundamentals and Applications (2019)

19. Versteeg, H.K., Malalasekera, W.: An Introduction to Computational Fluid Dynamics: The Finite, vol. Method. Pearson Education, London (2007)

20. Maza, D., Carvalho, M.S.: Trailing edge formation during slot coating of rectangular patches. J. Coat. Technol. Res. 14(5), 1003-1013 (2017). https://doi.org/10.1007/s11998017-9962-1

21. Matuttis, H.-G., Chen, J.: Understanding the Discrete Element Method: Simulation of NonSpherical Particles for Granular and Multi-Body Systems. Wiley, Hoboken (2014)

22. Violeau, D.: Fluid Mechanics and the SPH Method: Theory and Applications. Oxford University Press, Oxford (2012) 
23. Canuto, C., et al.: Spectral Methods: Evolution to Complex Geometries and Applications to Fluid Dynamics. Springer, Heidelberg (2007). https://doi.org/10.1007/978-3-540-30728-0

24. Oñate, E., Owen, R. (eds.): Particle-Based Methods: Fundamentals and Applications, vol. 25. Springer, Dordrecht (2011). https://doi.org/10.1007/978-94-007-0735-1

25. ParaView. https://www.paraview.org/

26. Kumar, S.: Object-oriented finite element programming for engineering analysis in $\mathrm{C}++$. JSW 5(7), 689-696 (2010)

27. SUNDIALS. https://computing.llnl.gov/projects/sundials

28. GSL. https://www.gnu.org/software/gsl/

29. Jacob, F., Ted, B.: A First Course in Finite Elements. Wiley, Hoboken (2007)

30. Cengel, Y.A.: Fluid Mechanics. Tata McGraw-Hill Education, New York (2010)

31. Gabbanelli, S., Drazer, G., Koplik, J.: Lattice Boltzmann method for non-Newtonian (powerlaw) fluids. Phys. Rev. E 72(4), 046312 (2005)

32. Wang, C.-H., Ho, J.-R.: A lattice Boltzmann approach for the non-Newtonian effect in the blood flow. Comput. Math. Appl. 62(1), 75-86 (2011)

33. Wang, C.Y.: Exact solutions of the steady-state Navier-Stokes equations. Ann. Rev. Fluid Mech. 23(1), 159-177 (1991)

34. Shankar, P.N., Deshpande, M.D.: Fluid mechanics in the driven cavity. Ann. Rev. Fluid Mech. 32(1), 93-136 (2000)

35. Adriasola, J.M., Janssen, R.H.A.: Best practices for design of slurry flow distributions (2018)

36. Abulnaga, B.E.: Slurry Systems Handbook. McGraw-Hill, New York (2002) 